\title{
Characterization of cerebral microbleeds and microinfarcts in patients with spontaneous intracerebral hemorrhage: a 7T MRI and histopathology study
}

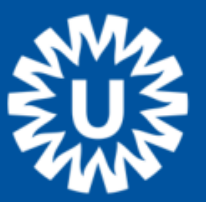

\section{UMC Utrecht}

Brain Center Rudolf Magnus

Wilmar MT Jolink ${ }^{1}$ Susanne J van Veluw², Jaco JM Zwanenburg ${ }^{3}$, Annemieke J Rozemuller ${ }^{4,5}$, Wim van Hecke ${ }^{4}$, Matthew P Frosch ${ }^{6}$, Brian J Bacskai ${ }^{7}$, Steven M Greenberg ${ }^{2}$, Catharina JM Klijn ${ }^{1,}$

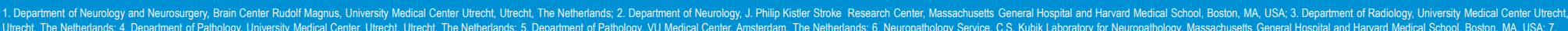

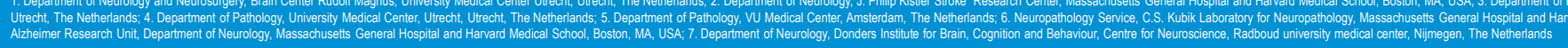

\section{Background}

- Spontaneous intracerebral hemorrhage $(\mathrm{ICH})$ is caused by hypertensive vasculopathy (deep ICH) and cerebral amyloid angiopathy (lobar ICH in elderly patients) $\rightarrow$ insufficient simplicifation

- Manifestations of small vessel disease (SVD) are increasingly recognized as potential factors in understanding the underlying vascular disease, for example: Cerebral microbleeds (CMB)

Ischemic lesions: acute (DWI) and chronic (cortical microinfarcts (CMI))

- Histopathology of MR-observed CMls and CMBs are heterogeneous

Aim

- To gain insight in the underlying small vessel disease in patients with spontaneous $\mathrm{ICH}$ using a combination of post-mortem 7 tesla (7T) MRI and histopathology of focal microvascular lesions

\section{Methods}

- From the biobank of the UMC Utrecht and the Netherlands Brain Bank we selected 20 patients (12 women, 8 men) who died as a consequence of $\mathrm{ICH}$ (median age 77 years; 8 deep, 9 lobar, 3 infratentorial)

- From each case, we scanned three formalin-fixed coronal slices with 7T MRI using high resolution $\mathrm{T} 2$ and $\mathrm{T}^{*}$-weighted sequences

- Acquired images were screened for cerebral microbleeds (CMBs) and cortical microinfarcts (CMls)

- From each case we sampled at least one $\mathrm{CMB}$ and one $\mathrm{CMI}$ and processed the samples for histopathological analysis

- We used standard hematoxylin and eosin (H\&E) stain to identify the lesions on the first sections stained adjacent sections for amyloid $\beta$, iron and fibrin to further characterize the lesions
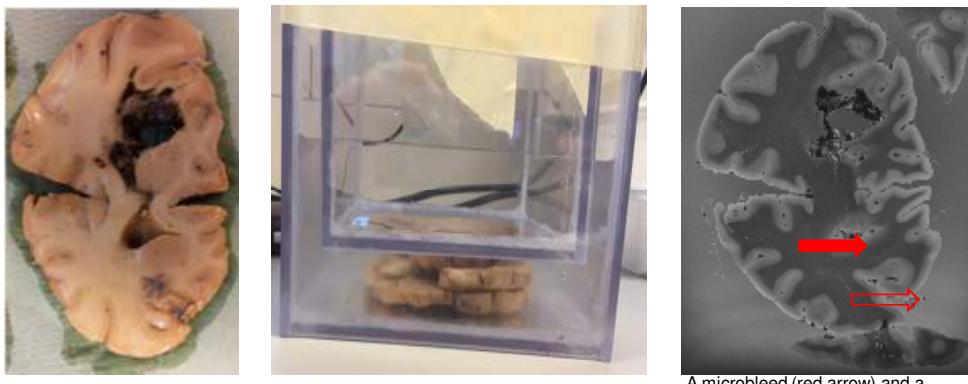
A microbleed (red arrow) and a observed on a T2*-weighted MRI.
Results

- 7T MRI identified lesions:

$$
\begin{aligned}
& \text { - } 132 \text { CMls } \\
& \text { o } 204 \text { CMBs }
\end{aligned}
$$

- 4 cases without lesions
- SCAA
- Deep ICH
- Cerebellar hemorrhage
- Pons hemorrhage

- Sections

- 28/72 lesions confirmed on H\&E

- 21/42 CMBs

- 7/30 CMls

- 36 additional lesions

- 18 in retrospect found on MRI

- Lesions in retrospect not verified on MRI mostly CMls and acute CMBs

- 44 lesions not found

7T MRI identified lesions in non-lobar ICH patients
\begin{tabular}{|l|l|l|l|l|l|l|l|l|l|l|l|l|l|}
\hline Case no. MRI identified lesions in lobar ICH patients \\
\hline 1 & Diagnosis & Deep, HTN & F & 49 & 1 & 5 & 9 & HCHWA-D & M & 56 & 29 & 7 \\
\hline 2 & Deep, possible HTN & F & 44 & 0 & 1 & 10 & SCAA & M & 76 & 40 & 1 \\
\hline 3 & Deep, HTN & M & 46 & 23 & 2 & 11 & No SCAA & F & 78 & 3 & 3 \\
\hline 4 & Pons, HTN en OAC & F & 75 & 3 & 0 & $12^{*}$ & SCAA & M & 85 & 1 & 38 \\
\hline 5 & Deep, HTN & F & 85 & 3 & 7 & 13 & SCAA & M & 78 & 0 & 0 \\
\hline 6 & Deep, HTN & M & 81 & 9 & 1 & 14 & SCAA & F & 83 & 66 & 8 \\
\hline 7 & Deep, HTN & F & 89 & 8 & 1 & 15 & SCAA & F & 81 & 54 & 1 \\
\hline 8 & Deep, HTN & F & 89 & 2 & 0 & 16 & SCAA & F & 73 & 1 & 16 \\
\hline
\end{tabular}

CMBS = cerebral microbleeds, $C M I s=$ cortical microinfarcts, $H C H W A-D=$ hereditary cerebral hemorrhage with amyloidosis Dutch type, $\mathrm{HTN}=$ hypertension, $\mathrm{OAC}=$ oral anticoagulants, SCAA = sporadic cerebral amyloid angiopathy

\section{Sections}

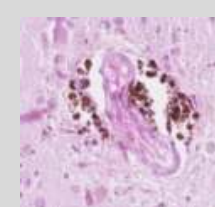

$H \& E$

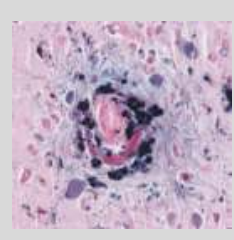

Iron

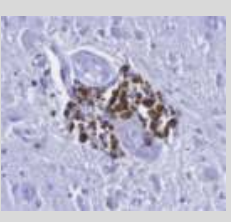

Amyloid $\beta$

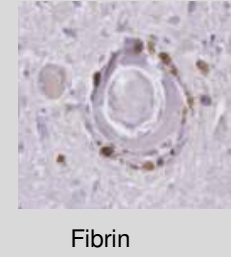

Fibrin
Example of a CMB in a 56 years old man with HCHWA-D

\section{Conclusion}

- $\mathrm{CMBs}$ and CMls are more prevalent in lobar $\mathrm{ICH}$ compared to non-lobar ICH

- CMBs are histologically heterogeous lesions and mostly not visible on MRI in the acute stage

Around CMBs we found more leakage of iron and fibrin compared to CMls

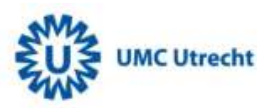

